\section{Infected urachal cyst in the adult}

\author{
Ricardo Borges, Paulo Temido, \\ Vânia Grenha, Bruno Pereira, \\ Ricardo Leão, Pedro Peralta, \\ Hugo Coelho, Ricardo Godinho, Luis \\ Sousa, Paulo Azinhais, Edson Retroz, \\ Álvaro Brandão, Fernando Sobral \\ Department of Urology, Centro \\ Hospitalar de Coimbra, EPE, Portugal
}

\begin{abstract}
Infected urachal cysts are a rare clinical manifestation in adults. We present the case of an adult male patient with an infected urachal cyst, discuss the embryology, clinical presentation, diagnostics and the therapeutic procedure. A high index of suspicion is necessary to diagnose this condition with unspecific clinical features. Ultrasound and MRI are useful in making the diagnosis. Surgery continues to be the therapeutic procedure of choice.
\end{abstract}

\section{Introduction}

In 1550 , the first reported urachal anomaly was described by Cabriolus in a patient who simultaneously voided from her umbilicus and urethra. ${ }^{1}$ Urachal patency results from a failure of the obliterative embryologic process occurring in the fourth month of gestation. With infrequent and variable presentations, diagnosing urachal anomalies can be chalenging. ${ }^{2}$ Urachal anomalies are usually seen in children and are rare in adults. ${ }^{3}$ The clinical manifestations may mimic many intraabdominal or pelvic disease processes. ${ }^{4}$ In the past, the definite diagnosis was frequently made at the time of surgery. Understanding the anatomy and imaging features of urachal anomalies is important for correct diagnosis and treatment. The authors present a case of an infected urachal cyst in adulthood.

\section{Case Report}

A 24 year-old caucasian male was referred to the Urology department with a 5 day history of hypogastric pain, polachyuria and subfebril temperature. The pain radiated to the penile glans and worsened when he strained to urinate or defecate. He gave no history of backpain, haematuria, nausea, vomiting, change in bowel habit, umbilical discharge or previous similar episodes. Systemic review revealed no abnormality. He already started a course of ciprofloxacin, without relief. On examination, he had a temperature of $37^{\circ} \mathrm{C}$ and was haemodinamically stable. Abdominal examination revealed a tender midline infraumbilical mass, without evidence of rebound tenderness. Haematology showed leucocytosis $(13,100 / \mu \mathrm{L})$ with neutrophylia (79.4\%) and raised CReactive Protein $(13.4 \mathrm{mg} / \mathrm{dL})$, with normal urine sediment and negative urine culture tests. Abdominal ultrasound scan showed a 3 $\mathrm{cm}$ echogenic collection, surrounded by a thick (6 mm) irregular wall. An MRI scan confirmed the diagnosis of urachal cyst not contiguous with the bladder or bowel wall (Figure 1). With the presumptive diagnosis of an infected urachal cyst, the initial management was administration of broad-spectrum intravenous antibiotic therapy (Meropenem) and ultrasound-guided puncture with drainage of a thick bloody fluid and placement of a pigtail drain. The cultures obtained revealed positive for Streptococcus anginosus. Cytological study was negative for neoplastic cells. The radiographic contrasted study of the cyst and bladder excluded any fistulous communication. With the infection subsided, he was submitted to complete excision of the cyst, urachal remnants and a cuff of bladder (Figure 2). Histopathological analysis of the resected specimen showed chronic inflammation with no evidence of malignancy. Recovery was unremarkable.

\section{Discussion}

\section{Embriology}

A comprehensive understanding of the anatomy and embryologic development of the bladder and urachus is mandatory to correctly interpret the urachal anomalies. Between the $4^{\text {th }}$ and $6^{\text {th }}$ weeks of gestation, the urorectal septum divides the endodermal cloaca into a ventral urogenital sinus and a dorsal rectum (Figure 3). The cranial part of the urogenital sinus is continuous with the allantois and develops into the bladder and pelvic urethra. Around the $4^{\text {th }}$ to the $5^{\text {th }}$ month of gestation, the allantoic duct and the ventral cloaca involute as the bladder descends into the pelvis. The descent causes the allantoic duct to elongate because it does not grow with the embryo. This epithelialized fibromuscular tube continues to become narrower until it is obliterated into a thick fibrous cord, the urachus. The obliterated urachus becomes the median umbilical ligament and connects the apex of the bladder with the umbilicus. ${ }^{5}$ It lies between the transverse fascia and the parietal peritoneum, where it is compartmentalized by the umbilicovesical fas-
Correspondence: Ricardo Borges, Department of Urology, Centro Hospitalar de Coimbra, EPE, Quinta dos Vales, 3041-801 S. Martinho do Bispo, Coimbra, Portugal.

E-mail: rlgborges@gmail.com

Key words: urachal cyst, embryologic process, ultrasound.

Received for publication: 13 April 2011. Accepted for publication: 30 June 2011.

This work is licensed under a Creative Commons Attribution NonCommercial 3.0 License (CC BYNC 3.0).

(C) Copyright R. Borges, 2011

Licensee PAGEPress, Italy

Urogynaecologia 2011; 25:e3

doi:10.4081/uij.2011.e3

cia. Disease processes usually remain contained inside this pyramid-shaped space.

\section{Urachal anomalies}

When the urachus fails to regress, it can remain completely open or obliterate partially, leading to the formation of cystic structures at any site throughout its course. Four different urachal anomalies have been described (Figure 4). In order of frequency, they are: ${ }^{6} \mathrm{i}$ ) Patent urachus (50\%): represents a persistent communication between the bladder and the umbilicus, suspected in the neonatal period by continuous or intermittent drainage of fluid from the umbilicus. Additional presentations include an enlarged or edematous umbilicus and delayed healing of the cord stump. ${ }^{6}$ ii) Urachal cyst (30\%): there is no communication of the cyst with the bladder or umbilicus. However, the fluid-filled cyst can drain through the umbilicus or into the bladder intermittently. Urachal cysts are found more commonly in the distal part of the urachus owing to epithelial desquamation and degeneration. ${ }^{7,8}$ iii) Umbilical-urachus sinus (15\%): the urachus obliterates at the bladder level but remains open at the umbilical site, causing a continuously draining sinus. The presentation is similar to that of the patent urachus. The diagnosis is made by sinogram. ${ }^{6}$ iv) Vesicourachal diverticulum (3-5\%): the urachus obliterates almost completely, except at the level of bladder apex. Here it forms a diverticulum of varying size. These lesions are usually nonsymptomatic and found incidentally on nonrelated radiographic workups. ${ }^{6}$

\section{Diagnosis}

Urachal cysts present in a variety of clinical presentations including recurrent urinary tract infections, macroscopic hematuria, hypogastric midline tenderness often associated with a 


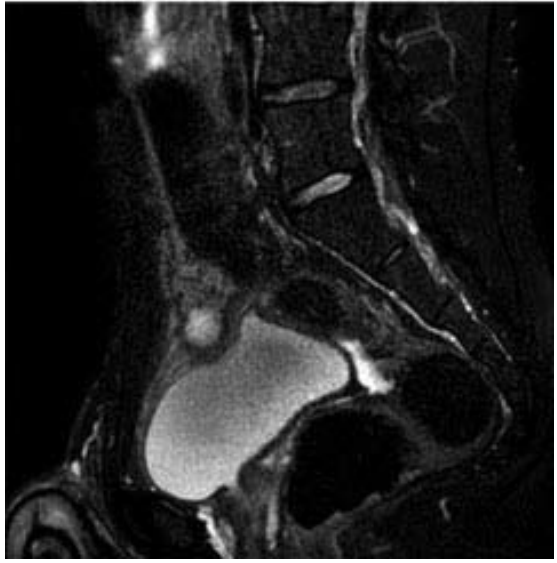

Figure 1. Magnetic resonance imaging scan showing high signal fluid within the urachal remnant cyst.

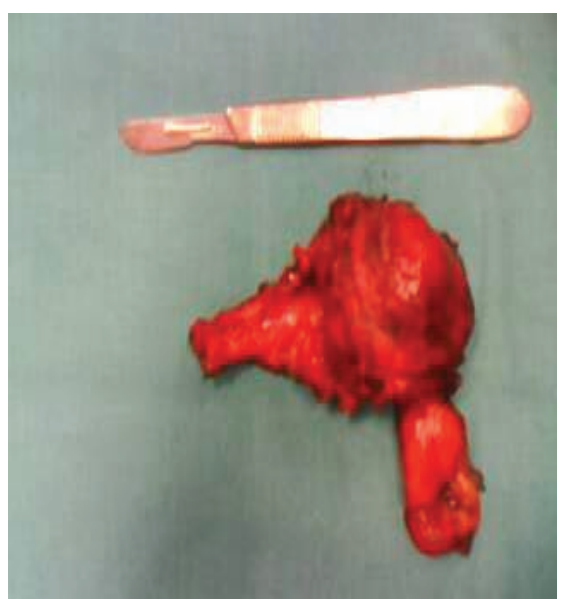

Figure 2. Surgical specimen.

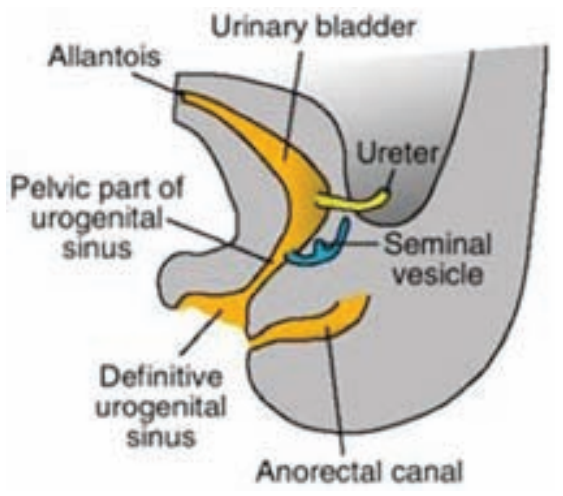

Figure 3. Development of the urinary bladder (adapted from Langman's embryology $\left.10^{\text {th }} \mathrm{ed}^{5}\right)$.
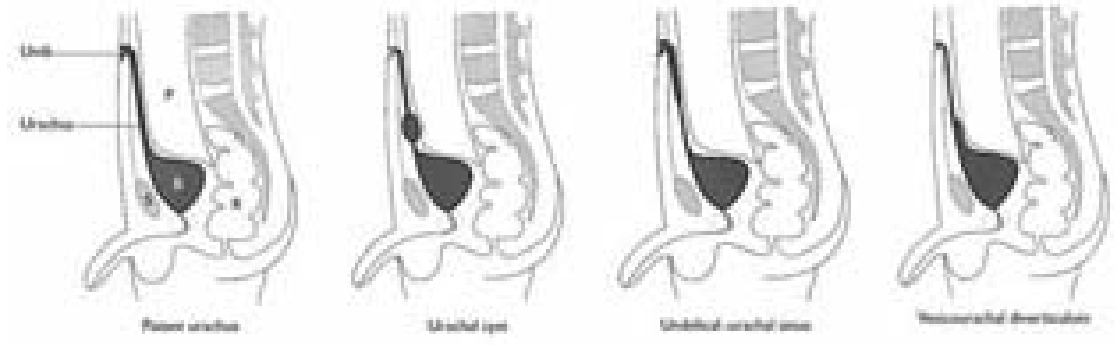

Figure 4 - Urachal anomalies. (adapted from Yoo et al..$\left.^{21}\right)$.

mass, umbilical discharge, and even peritonitis. In children a urachal cyst is the common presentation while in adults infected urachal sinuses are seen more frequently. ${ }^{9}$ Morbidity is related to infection and late occurring malignant changes. When grossly infected, the cyst often expands. This pyourachus can then establish communication with the umbilicus, bladder, bowel $^{7,10}$ or rupture intraperitoneally. ${ }^{11,12}$ Bacteria commonly found in descending order include Staphylococcus aureus, Escherichia coli, Enterococcus, and Citrobacter and, rarely, Proteus species. ${ }^{13}$ The penetration route is discussible (umbilical the most frequent). The pathogenesis of urachal carcinoma is poorly understood. Adenocarcinoma is believed to arise from malignant transformation of columnar metaplasia in as many as $84 \%$ of patients. However, in $3 \%$ it arises from metaplastic conversion of transitional cell epithelium to glandular epithelium. About $70 \%$ of urachal carcinoma is mucin producing adenocarcinoma and 15\% are non-mucin producing adenocarcinoma. Incidence of transitional cell carcinoma arising from remnant of transitional epithelium is low. $75 \%$ of urachal neoplasms in patients less than 29 years of age are sarcomas. Rarely, squamous cell carcinoma can also occur. ${ }^{14}$ Most (90\%) of urachal carcinomas are juxtavesical, specifically supravesical, anterosuperior to the bladder or in the midline. ${ }^{14}$ Intracystic lithiasis is a rare complication, frequently asymptomatic and diagnosed by routine abdominal ultrasound. ${ }^{15} \mathrm{~A}$ high degree of clinical suspicion followed by an ultrasound has been shown to be the most effective way of diagnosing a urachal cyst or abscess. Diagnostic findings include a midline, cystic, extraperitoneal swelling located between the bladder and the umbilicus. Sinography is useful to delineate the urachal sinuses. Abdominal CT or MRI can detect the pyourachus through imaging a mass located deep to the rectus abdominis between the bladder and the umbilicus with a conical shape, peripheral inflammatory changes in the surrounding tissues, and intraperitoneal fluid. Retrograde cystography is helpful in characterizing bladder patency. Cistoscopy can complete the diagnostic workup to evaluate bladder wall mucosal integrity. ${ }^{15}$

\section{Treatment}

Management of an infected urachus with abscess formation includes initial drainage under antibiotic coverage, followed by complete excision of the umbilicovesical tract including a cuff of bladder once the infection has subsided. ${ }^{16}$ For lesions not communicating with the bladder, conservative excision of the remnant cyst is adequate. Removal of all urachal elements is necessary to avoid recurrence of the cyst infection or occurrence of adenocarcinoma of the unresected or incompletely resected remnant. ${ }^{17}$ Traditionally excision of the urachus starts at the umbilicus and extends down to the bladder through an extra-peritoneal approach. More recently laparoscopic excision of the urachal remnant has been proposed to be as effective and as safe as the open operation with the additional advantages of decreased hospital stay, analgesic requirement, and convalescence. ${ }^{18-20}$ The prognosis in cases of urachal carcinoma is worse than that of primary bladder carcinoma because the tumour arises outside the bladder and involves the mucosa late in the disease process. Local invasion frequently presents before diagnosis. ${ }^{21}$

\section{Conclusions}

Urachal anomalies are uncommon and the literature is primarily comprised of case reports. Conclusions regarding the presentation and diagnosis of these anomalies can only be elucidated by reviewing a large experience of cases. In the adult population urachal anomalies are a rare cause of abdominal pain and present in a variety of ways. Through clinical suspicion and appropriate radiographic studies the correct diagnosis can be made. The urologist must be familiar with the presentation and management of this rare condition. We recommend a 2 stage treatment with a combination of broad spectrum antibiotics, puncture and drainage, followed by interval excision after resolution of sepsis. ${ }^{22}$ 
Urachal anomalies: defining the best diagnostic modality. Urology 1998;52:120-2.

9. Iuchtman M, Rahav $\mathrm{S}$, Zer $\mathrm{M}$, et al. Management of urachal anomalies in children and adults. Urology 1993;42: 426-30.

10. Rapoport D, Ross A, Goshko V, McAuley I. Urachal-sigmoid fistula associated with diverticular disease. Can Urol Assoc J 2007;1: 52-4.

11. Horgan PG, Johnson S, Courtney D. Intraperitoneal rupture of an infected urachus. Br J Urol 1994;73:216.

12. Chen TF, Collier DS. Intraperitoneal rupture of an infected urachal cyst in an infant. Case report. Eur J Surg 1991;157:67-8.

13. Mesrobian HG, Zacharias A, Balcom AH, Cohen RD. Ten years of experience with isolated urachal anomalies in children. J Urol 1997;158:1316-8.

14. Soni SP, Shah SR, Patel H, Patel D. Case report: Urachal pathologies. Ind J Radiol Imag 2005;15:357-60.

15. Arifa IH, Khadraoui H, Mhiri M. Atlas d'imagerie de l'ouraque du normal au pathologique. Tunisie; 2004.
16. Ward TT, Saltzman E, Chiang S. Infected urachal remnants in the adult: case report and review. Clin Infect Dis 1993;16:26-9.

17. Goldman IL, Caldamone AA, Gauderer M, et al. Infected urachal cysts: a review of 10 cases. J Urol 1988;140:375-8.

18. Chiarenza SF, Scarpa MG, D'Agostino S, et al. Laparoscopic excision of urachal cyst in pediatric age: report of three cases and review of the literature. J Laparoendosc Adv Surg Tech A 2009;19:S183-6.

19. Pust A, Ovenbeck R, Erbersdobler A, Dieckmann KP. Laparoscopic management of patent urachus in an adult man. Urol Int 2007;79:184-6.

20. Stone NN, Garden RJ, Weber H. Laparoscopic excision of a urachal cyst. Urology 1995; 45:161-4.

21. Ashley RA, Inman BA, Sebo TJ, et al. Urachal carcinoma: clinicopathologic features and long-term outcomes of an aggressive malignancy. Cancer 2006;107:712-20.

22. Yoo KH, Lee SJ, Chang SG. Treatment of infected urachal cysts. Yonsei Med J 2006;47: 423-7. 